\title{
Die elastische und inelastische Streuung von negativen K-Mesonen an freien Protonen bei Energien bis $90 \mathrm{MeV}$.
}

\author{
H. GöING \\ Max-Planck-Institut für Physik und Astrophysik - München \\ Nuovo Cimento, 16, $848(1960)$
}

\section{A D D E N D U M}

In der genannten Arbeit wurde erwähnt, daß 55\% der wom Verfasser analysierten Ereignisse von den Instituten in Bologna, Paris un Parma beigesteuert worden waren; es war jedoch versehentlich der Hinweis darauf unterlassen worden, daß die analysierten Ereignisse zum größten Teil mit denen identisch sind, die auch in der Gemeinschaftsarbeit von Alles et al. (1) behandelt wurden. Der Verfasser dankt seinen Kollegen in Bologna, Paris, Parma und Vünchen für ihre Hilfe und Mitarbeit.

(1) W. Altes, N. N. Biswas, M. Ceccarelli, R. Gessaroli, G. Qunkent, H. Göoing, K. Gottstein, W. Püschel, J. Tietge, G. T. Zorn, J. Crussard, J. Hennessy, G. Dascola und S. MoRa: Nuovo Cimento, 11, 771 (1959). 